\title{
A Combined Model of Congestion Toll Pricing Based on System Optimization with Minimum Toll
}

\author{
Hamid MIRZAHOSSEIN, Shahriar Afandizadeh ZARGARI
}

\begin{abstract}
Preventing the congestion is one of the main concerns of traffic managers and urban planners around the world. In response to this matter in transportation sector, planners have suggested toll pricing policies. This paper presents a combined optimization model as a new method to estimate the potential combination of travel time, and congestion toll that is implemented in a nine-node transportation network or Hearn network. This approach works as an urban travel demand management (UTDM) policy that imposes the cost of travel to travelers by calculating the marginal cost (MC), but the introduced model is optimized by minimizing the combination of travel time as an example of average cost (AC) and congestion toll as an example of MC simultaneously. Results show the total amount of flow is increased to 344,183 and the total amount of $\mathrm{MC}$ is decreased to 534,522 in comparison with the previous models.
\end{abstract}

Keywords: combined model; congestion; marginal cost; minimization; travel time; Urban Travel Demand Management (UTDM)

\section{INTRODUCTION}

Due to population growth in cities and the health care arguments, demand and willingness to use private vehicles (PV) has increased. Since the network expansion could not support all the PV demand and moreover based on the Braess's paradox [1] it is not the best solution most of the time. Thus, the demand of using the network becomes greater than the supply and this issue makes disequilibrium in traffic network. In this regard, the congestion is the result of this situation that causes various mental and environmental issues. Traffic congestion is an inherently challenging concept resulting in incrementally higher delays, pollution emissions, fuel waste and non-predictable travelling times [2]. Evaluation shows that the cost of congestion in 2010 for urban transportation network of the USA is about USD 101 billion [3, 4].

Inhibiting the congestion in urban transportation networks is one of the main concerns of traffic managers around the world. In response to this matter, they try to describe a stable condition for urban traffic network. Although, some studies develop microscopic aspect of this issue by changing and refining traffic lights schedules during congestion periods using internal/external traffic metering strategy [5], but these kinds of tactics just can prove to be beneficial for a short term or for specific location of a sub-network with special situation under specific conditions. The main causes of congestion and obstruction of networks are a lack of equilibrium and unstable relationship between the supply and demand of transportation; however, increasing the supply lonely could not solve the issue as a long-term solution. Nowadays, traffic experts suggested travel demand management (TDM) as a sustainable solution to control the demand for using the transportation infrastructure more efficiently. TDM strategies do not encourage decision makers to expand and widen the roads and highways as a solution for managing the traffic congestion. They try to manage the demand to use the provided supply more efficiently. Congestion toll pricing is one of the most effective methods in travel demand management (TDM) which has big advantages over the other TDM policies through encouraging the users and systems to adjust all aspect of their behaviour: number of trips, destination, mode of transport, time of day, route, and so on [6]. In this paper, congestion toll pricing is considered as a TDM policy for increasing the total flow and decreasing the total hidden cost or marginal cost (MC) in transportation network.

Some researches show that people do not want to pay for something from which they want to earn, not for something that they do not want to exist, like congestion [7]. Moreover, people do not want to pay for something they used before as a free service [8]. Although this may be true, but Guzman et al. [9] find a new method by dynamic simulation to measure the real benefits of each person in the short, medium, and long term due to pricing, by considering socio-economic efficiency and environmental criteria to optimize a cordon tolls scheme. Theory and practice have shown that receiving tolls, when designed and determined properly, can play a great role in reducing traffic congestion [10]. In other words, imposing toll on transportation network can help users to decrease urban traffic congestion and its negative effects such as total lost time, environmental pollution and so on.

In fact, by determining optimum and appropriate tolls, passengers think whether their new total travel costs which consist of their travel time and their MC are still affordable or not. Then they will either use network links with congestion toll and pay the difference of MC from $\mathrm{AC}$, or like the second part of users not use tolled links. In this regard, this paper presents a combined model that considers the minimization of travel time and congestion toll simultaneously in one objective function. For better determination of results, the proposed model is implemented in a well-known network that is used as a testbed in many studies in the field of congestion toll pricing.

\section{BRIEF LITERATURE REVIEW}

Congestion toll pricing on transportation networks is considered as a traditional issue in traffic management systems. During the last decades, several studies have been performed by economists and transportation planners [11] to achieve two main objectives. The first one is reducing or avoiding the congestion, and the second one is funding for implementation and maintenance of transportation projects. Most of the economists argue that the solution of 
any supply-demand imbalance is in the cost and pricing mechanism [12]. In this regard, various methods have been proposed for collecting the tolls. Pigou [13], one of the pioneers, offered a tax model and proposed the concept of marginal social cost pricing. Later, Knight [14] expanded the tax model based on the drivers' route choice behaviour. Nevertheless, most of the applied toll pricing models are based on the equilibrium condition in network, firstly proposed by Wardrop [15]. Wardrop, introduced two principles, i.e. User Equilibrium (UE) and System Optimum (SO), which represent the fundamentals of traffic assignment. Later, Beckmann et al. [16] proposed the first practical formulation for static traffic assignment using the principles of equilibrium. The readers are referred to [17] for more background about these kinds of traffic assignment problems. In static traffic assignment, the traffic flows are determined based on minimum cost between each origin-destination (OD) pairs. Also, the demand is constant and assigned to links based on systembalancing constraints. It means users have many choices, and they actually decide according to their economic situation. In this case, tolls are imposed on users to change the traffic flow pattern from UE to SO. In fact, the tolls are determined in a way that results in the possibility or impossibility of trip generation, shifting the trip from the peak to off-peak hours, or choosing the routes by users. Collecting tolls makes the users paying for negative effects of their presence in congested areas [18]. Thus, by assessing a proper toll on each link, the traffic flow situation will be close to SO in terms of minimizing the total travel cost. Hence, congestion pricing recommends charges that force users to adopt these externalities to some extent, thus reducing or eliminating the deadweight loss [19].

In order to determine proper tolls on each of the network links, different mathematical pricing models are presented in the literature [20-24]. These models adopt a framework for congestion toll pricing while different objectives are considered to calculate the marginal cost of congestion. The objectives are eventuated by some constraints that are imposed on the problem and the tolls are the decision variables. Some of the most famous ones are MinSys, MinMax, and MinTB models [20-22, 24, 25]. The models have been presented as a two stage optimization problem that uses the arc-node formulation of feasible flows [21, 24]. At first, the traffic assignment is solved based on system optimum; secondly, tolls are assigned to the network links. In such problems, tolls usually have dimension of time, which means that the value of time (VOT) is equal to one. This assumption is extensively adopted for comparison purposes between different solution methods. Moreover, this is not an unrealistic assumption, for example Lan Jiangand Hani S. Mahmassani [26] tried to compare heterogeneity of user preference in forecasting the demand for tolled facilities resulting from user response to pricing schemes which deal with users' VOT. Their results show the continuous nature of the VOT distribution. In other studies, researchers try to compare their solutions. In this regard, they consider the simple and same VOT for better comparison between their models. For instance, in some studies [20, 21, 23, 24] VOT is considered equivalent to travel time.

In the two-step strategy, tolls are not the only choices available as the decision variables. Michael Parkinson and Tyrell Rockefeller [23] presented a model for traffic assignment that uses a two-level mathematical program in which decision variables are entered at high levels and user equilibrium traffic assignment is in the lower level. Decision variables may include wasted time in each link, signal timings, congestion toll level of the link, and the flow in each link.

Similar to the decision variables, different approaches can be considered for evaluating the objective function. Recently, Chira et al. [27] show the influence of using different number of routes in traffic assignment model as a multi-objective optimization problem. Their results show the performance of the multi-objective evolutionary models for solving the traffic assignment problem. In another approach, Zhang et al. [28] present a comparison and optimization of cordon and area pricing from the perspective of travel demand management. They found that cordon pricing mainly affects trips with destination inside of the central business district (CBD) and origins outside, while area pricing imposed extra cost on the trips with either origins or destinations in the CBD. However, despite all the efforts in the last few years about modelling the first and second best pricing, there is no general and optimized result for modelling of these pricing methods in one-step.

In the present study, the emphasis is placed on the modification of the two-step pricing approach employed in the previous studies. Here, a coupled (combined) method is presented which simultaneously determines the traffic assignment and congestion toll pricing. To do so, a new combined cost function is defined as objective function while the constraints implemented in the optimization process are similar to traditional methods. This new approach of pricing is extensively analysed against urban transportation network i.e., Hearn network structure. The results of the new approach are compared with those of basic methods, i.e., MinSys, MinMax, MinTB and MSCP and comprehensive analysis will be presented.

\section{METHODOLOGY}

In congestion pricing terminology, the average cost $(A C)$ and marginal cost $(M C)$ are presented as generalized cost $(G C)$. The marginal cost means the change in the total cost, when the generated amount of private cars increases one unit. In transportation, it is defined when a user imposes a noticeable additional cost on other users [18].

$$
T C=(A C)(Q)
$$

Where: $T C$ - total cost, $A C$ - average cost for each user, $Q$ - all users of the route.

Therefore, the marginal cost $(M C)$ is defined as follows:

$$
\begin{aligned}
& M C=\frac{\mathrm{d}(T C)}{\mathrm{d} Q}=\frac{\mathrm{d}[(A C)(Q)]}{\mathrm{d} Q}=\frac{(A C) \mathrm{d} Q}{\mathrm{~d} Q}+\frac{Q \mathrm{~d}(A C)}{\mathrm{d} Q} \\
& \rightarrow M C=A C+\frac{Q \mathrm{~d}(A C)}{\mathrm{d} Q}
\end{aligned}
$$

Therefore, the objective function in pricing models tries to optimize the flow based on optimization in combination of travel time and toll of congestion. In these models, like the mentioned models in literature review, the $\mathrm{MC}$ is imposed on the users according to VOT equivalent, 
which shows the value of one unit of time for users who pass the determined link. In these models, the weight of travel time and congestion toll is considered equal, since the objective of the research is just to show how to solve the congestion toll pricing problem simultaneously with traffic assessment without using the bi-level optimization.

For instance, the MinSys algorithm [20] aims to minimize the total tolls collected while constraining the toll vector to be nonnegative and it contains two sections as a bi-level minimization problem. In step 1 , the model solves the system optimum problem to obtain an optimal solution represented as $v^{*}$ by the following equations:

$\min : s(v)^{\mathrm{T}} v$

Subject to

$v=Z x$

$\hat{A} x=b$

$x \geq 0$

In step 2 , the algorithm goes to minimize $\beta$ over the polyhedron that is defined by the following inequalities:

$Z^{\mathrm{T}}\left(s\left(v^{*}\right)\right)+\beta \geq \hat{A}^{\mathrm{T}} \rho$

$\left(v^{*}\right)^{\mathrm{T}}\left(s\left(v^{*}\right)+\beta\right)=b^{\mathrm{T}} \rho$

$\beta \geq 0$

In above formulations, parameters are defined based on the following notation. The $g=(N, A)$ is a network with $N$ being the node and $A$ being the arc set. Here, $Z$ is the arcpath incidence matrix and $\hat{A}$ is the origin - destination $(\mathrm{O}-$ D) pair-path incidence matrix of $g, k$ is a set of commodities that "flow" in the network, $b(k)$ is a demand vector associated to each $k$ (commodity), $\beta$ is the toll vector, $x$ is the vector of individual flows, $x(k)$ is the commodity $(k)$ flow (variable) vector and the sum of all the commodity flow vectors became the aggregate flow vector denoted as $v$ and its cost is $s(v) . \rho$ is the linear programming (LP) duality parameter which describes Wardrop's first principle [20]. In addition, the system feasible flows are introduced as follows:

$$
\begin{aligned}
& v=\sum_{k} x(k) \\
& \hat{A} x(k)=b(k) \quad \forall k \in K \\
& x(k) \geq 0 \quad \forall k
\end{aligned}
$$

In addition, in regard of the presented formulation for MinSys, the other formulations of MinMax and MinTB are presented in Hearn and Ramana research as a base study for comparing these formulations [21]. The MinMax minimizes the maximum toll on the transportation network:

$\min z$

Subject to

$$
\begin{aligned}
& (\beta, \rho) \in W\left(v^{*}\right) \\
& z \geq \beta_{a} \quad \forall a \in A \\
& \beta \geq 0
\end{aligned}
$$

The MinTB minimizes the number of toll booths [24]. The formulation requires a positive constant $M$ that exceeds the largest toll and a vector $\boldsymbol{y}$ of binary variable $y_{a}$. The formulation is as follows:

$\min \sum_{a \in A} y_{a}$

Subject to

$$
\begin{aligned}
& (\beta, \rho) \in W\left(v^{*}\right) \\
& \beta_{a} \leq M z_{a} \quad \forall a \in A \\
& y_{a} \in\{0,1\} \\
& \beta \geq 0
\end{aligned}
$$

In above formulations, $y_{a}$ is a binary variable and $M$ is a big number that refers to a large number associated with the artificial variables. This condition is satisfied only when all the constraints (except non-negativity) are lessthan constraints with a positive constant on the right-hand side.

\subsection{Suggested Objective Function}

This section presents the model that has been developed in this research. The model is a multi-objective optimization problem and tries to find the link flows. Also, find the optimized MC cost of congestion. The objective function and constraints are presented in Eqs. (22) to (29).

$$
\begin{aligned}
& \operatorname{MinZ}=\sum_{r s} \sum_{k \in K_{r s}} \sum_{a}\left[\delta_{a k}\left(t_{a}\left(x_{a}\right)+\tau_{a}\right)\right] x_{a} \\
& \forall r s, k \in K_{r s}, r s, a \in A
\end{aligned}
$$

Subject to

$$
\begin{aligned}
& f_{k} * T T_{r s}=\sum_{r s} \sum_{k \in K_{r s}} \sum_{a}\left[f_{k} *\left(t_{a}\left(x_{a}\right)+\tau_{a}\right) \delta_{a k}\right] \\
& \forall r s, k \in K_{r s}, r s, a \in A \\
& T T_{r s} \leq \sum_{r s} \sum_{k \in K_{r s}} \sum_{a}\left[\left(t_{a}\left(x_{a}\right)+\tau_{a}\right) \delta_{a k}\right] \\
& \forall r s, k \in K_{r s}, r s, a \in A \\
& \sum_{k \in K_{r s}} f_{k}=\boldsymbol{q}_{r s} \quad \forall r s \\
& \sum_{r s} \sum_{k \in K_{r s}} \delta_{a k} f_{k}=x_{a} \quad \forall a \in A \\
& \tau_{a} \geq 0 \quad \forall a \\
& x_{a} \geq 0 \quad \forall a \\
& x_{k} \geq 0 \quad \forall k \in K_{r s}, r s
\end{aligned}
$$


The notation in mentioned equations comes as follows: $Z$ - combined objective function

$a$ - represents a link in the transportation network $x_{a}$ - flow in link $a$

$x_{a}^{C M}$ - flow in link $a$ based on the combined model

$t_{a}\left(x_{a}\right)$ - travel time in link $a$

$\tau_{a}$ - toll on link $a$

$k$ - feasible paths in the network

$f_{k}$ - flow in path $k$

$r s$ - origin-destination set in the network

$\boldsymbol{q}_{r s}$ - demand matrix between $r s$

$\delta_{k}=\left\{\begin{array}{l}1 \text { for each } a \in f_{k} \\ 0\end{array}\right.$

$T T_{r s}$ - travel time in the shortest path between $r s$ $k_{r s}$ - set of feasible paths between $r s$

This model has one multi-objective function described in Eq. (22). This equation minimizes the total hidden cost by considering the number of tolled links in user equilibrium condition. The constraints (23) and (24) are supplementary ones and cause that for each origindestination pair $r s$, only the routes $k \in k_{r s}$ which cost equal to the shortest route $u_{r s}$ have non-zero flow. The constraint (25) ensures that the demand of each pair of origindestination is satisfied, in other words, the summation of all flows in all pairs of origin-destination is equal to the demand of that origin-destination pair. The constraint (26) makes the aggregated flow in the links become equal to the optimized system flow. The constraints (27) and (28) show that the toll and flow obtained for each link in the set of the network links should be positive. The constraint (29) show the path flow for each OD should be positive.

By solving the model and finding, the answer based on the objective functions and the defined constraints, the requirements of first degree of optimality for the obtained answers are practically satisfied. Also, the Hessian matrix for controlling the requirements for second degree of optimality could be checked, and if the function is convex for all variables the hessian matrix of the model is positivedefinite, so, the unity of the answer is controlled. As is mentioned in previous literatures, in all symmetric matrices, like Hessian matrix, if and only if the Eigen values of the matrix are positive, we can assume it as a positive-definite matrix [29]. Due to the high volume, the calculations are not included in this section.

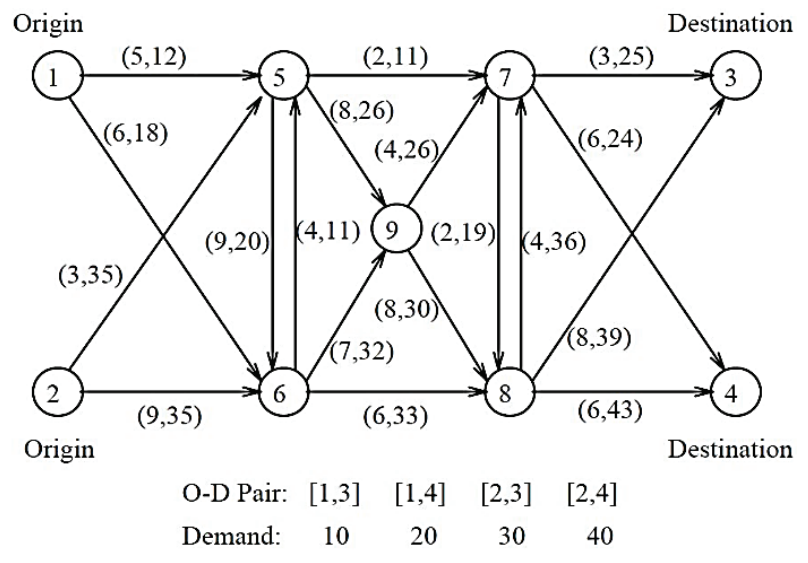

Figure 1 The nine node network [20-22, 24]

\section{NUMERICAL EXAMPLE}

This paper employed the nine-node network that is used in many researches $[20,21,23,24]$ as a testbed for providing a better comparison of the introduced model versus the previous ones that had solutions in congestion toll pricing optimization problem. By considering this issue, that the most studies and theoretical models are implemented based on this network, this network is considered for analysing and comparing the proposed model. The network topology is shown in Fig. 1.

As shown in Fig. 1, the network has nine nodes, eighteen links that have the same structure of cost function, and four pairs of origin-destination (OD) : $(1,3),(1,4),(2,3)$ and $(2,4)$. As shown in the bottom of Fig. 1, the demand of node one to node three equals 10 units; node one to node four equals 20 units; node two to node three equals 30 units, and node two to node four equals 40 units. The link time-travel $\left(t_{a}\right)$ or cost function structure is provided as follows:

$t_{a}=T_{a}\left[1+0.15\left(\frac{x_{a}}{C_{a}}\right)^{4}\right]$

The parameters $T_{a}$ and $C_{a}$ are constants values, which respectively represent free flow travel time and practical capacity of the links in the network, and their values are shown next to each link. For example, for the link (arc) from node 1 to 5 , the amount of describe parameters are $\left(T_{a}, C_{a}\right) \equiv(5,12)$ and so on for the other links.

Tab. 1 shows the results of running the model that is obtained by solving the combined model in Maple software for the nine-node network. This table has eight columns, which respectively from left to right include the link number used to number the 18 links based on the network topology. The next column, is "Arc"; it shows the start and end node of the links. The flow $x_{a}^{C M}$ column practically shows the flows obtained from the combined objective function, which means the flow is assigned based on the user decisions to find the minimum travel time by considering this assumption that they know traffic situation in long term. The travel time $t_{a}\left(x_{a}^{S O}\right)$ column shows the travel time in combined model from flow $x_{a}^{C M}$. The total travel time $x_{a}^{C M} * t_{a}\left(x_{a}^{S O}\right)$ column is calculated by multiplying the flow to travel time for each link. Toll $\tau_{a}$ column shows the calculation of tolls based on the combined congestion pricing model, and finally the total hidden cost $\tau_{a} * x_{a}^{C M}$ column is considered as the multiplication of the toll $\tau_{a}$ by flow $x_{a}^{C M}$.

As is shown in Tab. 1, the model finds the six links as potential links that would be considered for implementing the congestion toll pricing policy on them. In fact, these six links are the critical links in the network that the system should consider for controlling the demand on them. In other words, the network could be in an equilibrium condition if the system imposed the tolls on the introduced links as tolled links. Tab. 2 shows the results of the mentioned models in literature review for the nine-node 
network. By comparing the results of the previous models with the suggested model, the preference of the proposed model could be clear.

In addition, the combined model results show that the total cost is 3152,181 that covers the total travel time which is 2617,660 plus total hidden cost or MC which is 534,5215; in this regard, Tab. 3 and Fig. 2 summarize the final results of the model and also compare them with MSCP, MinSys, MinMax, and MinTB, since they have been used in many pricing studies with the same testbed. As it can be seen, not only is the total MC decreased in CM in comparison with the others, but also the flow of the combined model has been increased compared with the mentioned models.

Table 1 Results of the combined model for the nine-node network

\begin{tabular}{|c|c|c|c|c|c|c|c|}
\hline Link & Arc & $\begin{array}{l}\text { Flow } \\
x_{a}^{C M}\end{array}$ & $\begin{array}{l}\text { Travel time } \\
t_{a}\left(x_{a}^{C M}\right)\end{array}$ & $\begin{array}{l}\text { Total travel time } \\
x_{a}^{C M} * t_{a}\left(x_{a}^{C M}\right)\end{array}$ & $\begin{array}{c}\text { Toll } \\
\tau_{a}\end{array}$ & $\begin{array}{l}\text { Total marginal cost } \\
\qquad \tau_{a} * x_{a}^{C M}\end{array}$ & General cost (Total cost) \\
\hline 1 & $1-5$ & 13,8604 & 5,2837 & 87,8039 & 0,0000 & 0 & 87,8039 \\
\hline 2 & $1-6$ & 16,1396 & 7,5406 & 106,2265 & 3,6206 & 58,4358 & 164,6623 \\
\hline 3 & $2-5$ & 47,4885 & 3,6476 & 214,8892 & 0,8384 & 39,8165 & 254,7057 \\
\hline 4 & $2-6$ & 22,5115 & 9,9045 & 207,8048 & 0,0000 & 0 & 207,8048 \\
\hline 5 & $5-6$ & 0,0000 & 9,0000 & 0,0000 & 0,0026 & 0 & 0 \\
\hline 6 & $5-7$ & 28,9681 & 6,2202 & 475,9102 & 0,0000 & 0 & 475,9102 \\
\hline 7 & $5-9$ & 32,3808 & 9,2837 & 352,5276 & 0,0000 & 0 & 352,5276 \\
\hline 8 & $6-5$ & 0,0000 & 4,0000 & 0,0000 & 0,0000 & 0 & 0 \\
\hline 9 & $6-8$ & 26,8489 & 7,8425 & 171,6817 & 8,6948 & 233,4447 & 405,1264 \\
\hline 10 & $6-9$ & 11,8022 & 7,0267 & 82,8448 & 0,0000 & 0 & 82,8448 \\
\hline 11 & $7-3$ & 26,8400 & 3,8853 & 96,5657 & 6,9456 & 186,4188 & 282,9845 \\
\hline 12 & $7-4$ & 31,5843 & 6,5036 & 274,7666 & 0,0000 & 0 & 274,7666 \\
\hline 13 & $7-8$ & 0,0000 & 2,0000 & 0,0000 & 0,0000 & 0 & 0 \\
\hline 14 & $8-3$ & 13,1600 & 8,0060 & 105,4851 & 0,0000 & 0 & 105,4851 \\
\hline 15 & $8-4$ & 28,4157 & 6,6243 & 175,3716 & 0,0000 & 0 & 175,3716 \\
\hline 16 & $8-7$ & 0,0000 & 4,0000 & 0,0000 & 0,0000 & 0 & 0 \\
\hline 17 & $9-7$ & 29,4561 & 4,9366 & 146,9408 & 0,5534 & 16,3004 & 163,2411 \\
\hline 18 & $9-8$ & 14,7269 & 8,0158 & 118,8412 & 0,0071 & 0,1053 & 118,9465 \\
\hline \multicolumn{2}{|c|}{ Total } & 344,183 & 113,7211 & 2617,6597 & 20,6625 & 534,5215 & 3152,1811 \\
\hline
\end{tabular}

Table 2 Results of the MSCP, MinSys, MinMax, and MinTB models for the nine-node network that introduced in the literature review [20-22, 24, 25]

\begin{tabular}{|c|c|c|c|c|c|c|c|c|c|c|c|}
\hline \multirow[b]{2}{*}{ Arc } & \multirow[b]{2}{*}{$\begin{array}{c}\text { Flow } \\
x_{a}^{S O}\end{array}$} & \multirow[b]{2}{*}{$\begin{array}{c}\text { Travel } \\
\text { time } \\
t_{a}\left(x_{a}^{S O}\right)\end{array}$} & \multirow[b]{2}{*}{$\begin{array}{c}\text { Total travel } \\
\text { time } \\
x_{a}^{S O} * t_{a}\left(x_{a}^{S O}\right)\end{array}$} & \multicolumn{2}{|c|}{ MSCP } & \multicolumn{2}{|c|}{ MinSys } & \multicolumn{2}{|c|}{ MinMax } & \multicolumn{2}{|c|}{ MinTB } \\
\hline & & & & $\begin{array}{l}\text { Toll } \\
\tau_{a}\end{array}$ & $\begin{array}{c}\text { Total } \\
\text { marginal } \\
\text { cost } \\
\tau_{a} * x_{a}^{S O}\end{array}$ & $\begin{array}{l}\text { Toll } \\
\tau_{a}\end{array}$ & $\begin{array}{c}\text { Total } \\
\text { marginal } \\
\text { cost } \\
\tau_{a} * x_{a}^{S O}\end{array}$ & $\begin{array}{l}\text { Toll } \\
\tau_{a}\end{array}$ & $\begin{array}{c}\text { Total } \\
\text { marginal } \\
\text { cost } \\
\tau_{a} * x_{a}^{S O}\end{array}$ & $\begin{array}{l}\text { Toll } \\
\tau_{a}\end{array}$ & $\begin{array}{c}\text { Total } \\
\text { marginal cost } \\
\tau_{a} * x_{a}^{S O}\end{array}$ \\
\hline $1-5$ & 9,411 & 5,284 & 49,728 & 1,135 & 10,681 & 0,000 & 0,000 & 0,000 & 0,000 & 0,000 & 0,000 \\
\hline $1-6$ & 20,589 & 7,541 & 155,262 & 6,162 & 126,869 & 0,000 & 0,000 & 8,000 & 164,712 & 0,000 & 0,000 \\
\hline $2-5$ & 38,334 & 3,648 & 139,842 & 2,590 & 99,285 & 4,000 & 153,336 & 4,000 & 153,336 & 4,000 & 153,336 \\
\hline $2-6$ & 31,666 & 9,905 & 313,652 & 3,618 & 114,566 & 0,000 & 0,000 & 0,000 & 0,000 & 0,000 & 0,000 \\
\hline $5-6$ & 0,000 & 9,000 & 0,000 & 0,000 & 0,000 & 0,000 & 0,000 & 0,000 & 0,000 & 0,000 & 0,000 \\
\hline $5-7$ & 21,303 & 6,220 & 132,652 & 16,880 & 359,595 & 11,200 & 238,594 & 8,000 & 170,424 & 11,200 & 238,594 \\
\hline $5-9$ & 26,442 & 9,284 & 245,487 & 5,135 & 135,780 & 0,000 & 0,000 & 0,000 & 0,000 & 0,000 & 0,000 \\
\hline $6-5$ & 0,000 & 4,000 & 0,000 & 0,000 & 0,000 & 0,000 & 0,000 & 0,000 & 0,000 & 0,000 & 0,000 \\
\hline $6-8$ & 39,474 & 7,843 & 309,595 & 7,370 & 290,923 & 7,2 & 284,213 & 7,200 & 284,213 & 7,2 & 284,213 \\
\hline $6-9$ & 12,781 & 7,027 & 89,812 & 0,107 & 1,368 & 0,000 & 0,000 & 0,000 & 0,000 & 0,000 & 0,000 \\
\hline $7-3$ & 29,608 & 3,885 & 115,027 & 3,541 & 104,841 & 4,000 & 118,432 & 7,200 & 213,178 & 4,000 & 118,432 \\
\hline $7-4$ & 20,757 & 6,504 & 135,004 & 2,014 & 41,805 & 0,000 & 0,000 & 3,200 & 66,422 & 0,000 & 0,000 \\
\hline $7-8$ & 0,000 & 2,000 & 0,000 & 0,000 & 0,000 & 0,000 & 0,000 & 1,079 & 0,000 & 0,000 & 0,000 \\
\hline $8-3$ & 10,392 & 8,006 & 83,198 & 0,024 & 0,249 & 0,000 & 0,000 & 0,000 & 0,000 & 0,000 & 0,000 \\
\hline $8-4$ & 39,243 & 6,624 & 259,946 & 2,497 & 97,990 & 0,000 & 0,000 & 0,000 & 0,000 & 0,000 & 0,000 \\
\hline $8-7$ & 0,000 & 4,000 & 0,000 & 0,000 & 0 & 0,000 & 0,000 & 0,000 & 0,000 & 0,000 & 0,000 \\
\hline $9-7$ & 29,062 & 4,937 & 143,479 & 3,746 & 108,866 & 3,200 & 92,998 & 0,000 & 0,000 & 3,200 & 92,998 \\
\hline $9-8$ & 10,162 & 8,016 & 81,459 & 0,063 & 0,640 & 0,000 & 0,000 & 0,000 & 0,000 & 0,000 & 0,000 \\
\hline
\end{tabular}

$* x_{a}^{S O}$ - traffic flow based on the system optimum $(S O)$

Table 3 Summarized results of the combined model in comparison with the other studies

\begin{tabular}{|c|c|c|c|c|c|}
\hline Solutions & No. of tolled link & Total flow & Total marginal cost (MC) & Total travel time & Total cost \\
\hline MSCP & 14 & 339,2240 & 1493,458 & 2253,918 & 2253,918 \\
\hline MinSys & 5 & 339,2240 & 887,574 & 3141,492 \\
\hline MinMax & 7 & 339,2240 & 1167,572 & 2253,918 \\
\hline MinTB & 5 & 339,2240 & 887,574 & 2253,918 \\
\hline Combined model $(C M)$ & 6 & 344,18230 & 534,5215 & 314,490 & 2617,660 \\
\hline
\end{tabular}




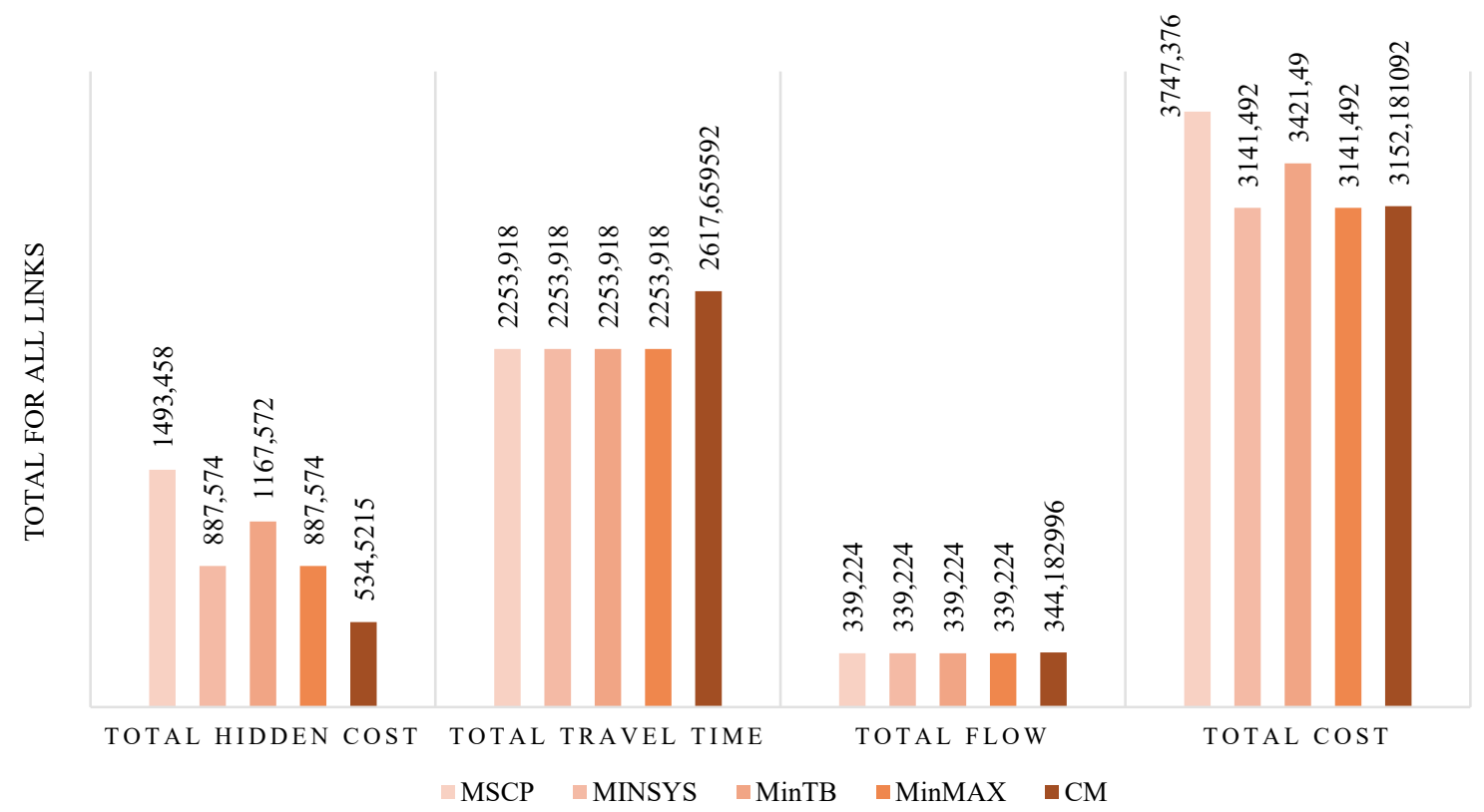

Figure 2 Total cost of suggested model in comparison with previous studies

\section{CONCLUSIONS}

A new approach which simultaneously considers the traffic assignment and congestion pricing has been introduced and extensively examined against other traditional methods. Implementing this approach in an urban transportaion network benchmark i.e., Hern structure, reveals that while the distribution of flow in the network links is reasonably close to the system equilibrium condition, the number of selected links for toll asignment is reduced.

For comparison purposes, the results of the present study are compared with MinSys, MinMax, MinTB and marginal-social-cost-pricing (MSCP) which were thoroughly studied in literature. Based on the comparisions made here, the following is concluded:

1) From the total-cost point of view, while the present model maintains a competitive price with respect to MinTB and MinSys, the Total flow of the network is increased and also, the total (hidden) marginal cost is decreased.

2) The present approach provides more realistic and practical pricing with respect to the traditional methods, which perform traffic assignment, and congestion pricing in segregated steps; i.e., here, the flow of the links is not considered as a predetermined constraint.

3) The advantages of the introduced model are achieved in the condition that the distribution of flow in each link is closely similar to system equilibrium condition. In other words, the equilibrium condition for a network can be reasonably satisfied.

4) Considering the flow of the links as a floating variable leads to more efficient network; i.e., higher total flow, lower marginal cost and total cost near the equilibrium condition.

\section{REFERENCES}

[1] Murchland, J. D. (1970). Braess's paradox of traffic flow. Transportation Research, 4(4), 391-394. https://doi.org/10.1016/0041-1647(70)90196-6

[2] Menelaou, C., Kolios, P., Timotheou, S., \& Panayiotou, C. G. (2016). A congestion-free vehicle route reservation architecture. Electrotechnical Conference (MELECON), $18^{\text {th }}$ Mediterranean, IEEE / Cyprus, 1-6. https://doi.org/10.1109/MELCON.2016.7495458

[3] Grant-Muller, S. \& Xu, M. (2014). The role of tradable credit schemes in road traffic congestion management. Transport Reviews, 34(2), 128-149. https://doi.org/10.1080/01441647.2014.880754

[4] Hensher, D. A. \& Bliemer, M. C. (2014). What type of road pricing scheme might appeal to politicians? Viewpoints on the challenge in gaining the citizen and public servant vote by staging reform. Transportation Research Part A: Policy and Practice, 61, 227-237. https://doi.org/10.1016/j.tra.2014.02.017

[5] Afandizadeh Zargari, S., Dehghani, N., Mirzahossein, H., \& Hamedi, M. (2016). Improving SCATS Operation during Congestion Periods Using Internal/External Traffic Metering Strategy. PROMET - Traffic \& Transportation, 28(1), 41-47. https://doi.org/10.7307/ptt.v28i1.1718

[6] De Palma, A. \& Lindsey, R. (2009). Traffic congestion pricing methods and technologies. Cahier de Recherche. HAL Id: hal-00414526, https://hal.archives-ouvertes.fr/hal00414526

[7] Thorpe, N., Hills, P., \& Jaensirisak, S. (2000). Public attitudes to TDM measures: a comparative study. Transport Policy, 7(4), 243-257. https://doi.org/10.1016/S0967-070X(00)00007-X

[8] Goodwin, P. \& Lyons, G. (2010). Public attitudes to transport: interpreting the evidence. Transportation planning and technology, 33(1), 3-17. https://doi.org/10.1016/S0967-070X(00)00007-X

[9] Guzman, L. A., Di Ciommo, F., \& Hoz, D. (2013). Integration of Congestion Pricing and Intertemporal Preference Rate in Social Welfare Function. Transportation Research Record: Journal of the Transportation Research Board, 2346(1), 1-12. https://doi.org/10.3141/2346-01

[10] Yildirim, M. B. (2001). Congestion toll pricing models and methods for variable demand networks. PhD Dissertation, University of Florida.

[11] Yang, H. \& Huang, H. J. (1998). Principle of marginal-cost pricing: how does it work in a general road network? 
Transportation Research Part A: Policy and Practice, 32(1), 45-54. https://doi.org/10.1016/S0965-8564(97)00018-9

[12] Bagloee, S. A., Asadi, M., \& Mohebbi, C. (2014). A Model for Screening Vulnerability in the Loan Market in the Context of Credit Rationing. International Journal of Strategic Decision Sciences (IJSDS), 5(1), 59-75. https://doi.org/10.4018/ijsds.2014010104

[13] Pigou, A. C. (1920). The Economics of Welfare, $4^{\text {th }}$. London: Macnillam.

[14] Knight, F. H. (1924). Some Fallacies in the Interpretation of Social Cost. Quarterly Journal of Economics, 38(4), 582606. https://doi.org/10.2307/1884592

[15] Wardrop, J. G. (1952). Some Theoretical Aspects of Road Traffic Research. ICE Proceedings: Engineering Divisions. Thomas Telford. https://doi.org/10.1680/ipeds.1952.11362

[16] Beckmann, M., McGuire, C. B., \& Winsten, C. B. (1956). Studies in the Economics of Transportation. Yale University Press.

[17] Florian, M. \& Hearn, D. (1995). Network equilibrium models and algorithms. Handbooks in Operations Research and Management Science, 8, 485-550. https://doi.org/10.1016/S0927-0507(05)80110-0

[18] Tezcan, H. O. (2009). Evaluating road pricing with an engineering perspective: aggregate and disaggregate analysis. Canadian Journal of Civil Engineering, 36(6), 1028-1036. https://doi.org/10.1139/L09-046

[19] Small, K. A. \& Verhoef, E. T. (2007). The economics of urban transportation. Routledge.

[20] Bergendorff, P., Hearn, D. W., \& Ramana, M. V. (1997). Congestion toll pricing of traffic networks. Network Optimization, Springer, 51-71. https://doi.org/10.1007/978-3-642-59179-2_4

[21] Hearn, D. W. \& Ramana, M. V. (1998). Solving congestion toll pricing models. Equilibrium and advanced transportation modelling, Springer, 109-124. https://doi.org/10.1007/978-1-4615-5757-9_6

[22] Hearn, D. W. \& Yildirim, M. B. (2002). A toll pricing framework for traffic assignment problems with elastic demand. Transportation and network analysis: Current trends, Springer, 135-145. https://doi.org/10.1007/978-1-4757-6871,-8_9

[23] Patriksson, M. \& Rockafellar, R. T. (2002). A mathematical model and descent algorithm for bilevel traffic management. Transportation Science, 36(3), 271-291. https://doi.org/10.1287/trsc.36.3.271.7826

[24] Yildirim, M. B. \& Hearn, D. W. (2005). A first best toll pricing framework for variable demand traffic assignment problems. Transportation Research Part B: Methodological, 39(8), 659-678. https://doi.org/10.1016/j.trb.2004.08.001

[25] Hearn, D. \& Ribera, J. (1980). Bounded flow equilibrium problems by penalty methods. Proceedings of IEEE International Conference on Circuits and Computers, 162166

[26] Jiang, L. \& Mahmassani, H. S. (2013). Toll Pricing: Computational Tests for Capturing Heterogeneity of User Preferences. Transportation Research Record: Journal of the Transportation Research Board, 2343(1), 105-115. https://doi.org/10.3141/2343-13

[27] Chira, C. \& Bazzan, A. L. (2015). Route assignment using multi-objective evolutionary search. Intelligent Computer Communication and Processing (ICCP), 2015 IEEE International Conference, 141-148. https://doi.org/10.1109//CCP.2015.7312619

[28] Zhang, L., Liu, H., \& Sun, D. (2014). Comparison and optimization of cordon and area pricings for managing travel demand. Transport, 29(3), 248-259. https://doi.org/10.3846/16484142.2014.914970

[29] Sheffi, Y. (1985). Urban transportation networks: equilibrium analysis with mathematical programming methods. Pretince Hall, Englewood Cliffs, NJ.

\section{Contact information:}

Hamid MIRZAHOSSEIN, Assistant Professor,

(Corresponding Author)

Department of Civil Engineering - Transportation Planning and Engineering, Faculty of Engineering and Technology, Imam Khomeini International University (IKIU),

34148 - 96818, Qazvin, Iran

E-mail: mirzahossein@eng.ikiu.ac.ir

Shahriar Afandizadeh ZARGARI, PhD, Associate Professor

Department of Transportation Planning and Engineering, School of Civil Engineering, Iran University of Science and Technology, Narmak, 16846-13114 Tehran, Iran

E-mail: zargari@iust.ac.ir 